\title{
RADIUS OF STARLIKENESS OF CONVEX COMBINATIONS OF UNIVALENT STARLIKE FUNCTIONS
}

\author{
D. H. HAMILTON ${ }^{1}$ AND P. D. TUAN
}

\begin{abstract}
The radius of starlikeness of the convex combination
\end{abstract}

$$
t f(z)+(1-t) g(z), \quad 0<t<1,
$$

where $f(z)$ and $g(z)$ are normalized univalent starlike functions, is $r_{u}=$ $0.4035 \ldots$, the positive root of the equation $r^{6}+5 r^{4}+79 r^{2}-13=0$.

Let $S$ denote the class of functions that are normalized and univalent in the unit disk $E=\{z:|z|<1\}$. Let St, $K$ be the subclasses of $S$ of functions that map $E$ onto starlike and convex domains respectively. Robertson [3] showed that the radius of univalence of the class

$$
F=\{h(z)=t f(z)+(1-t) g(z): f(z), g(z) \in \mathrm{St}, 0<t<1\}
$$

is $r_{u}$, where $r_{u}=0.4035 \ldots$ is the positive root of the equation $r^{6}+5 r^{4}+$ $79 r^{2}-13=0$. Labelle and Rahman [2] found that for $f, g \in \operatorname{St}(K)$, the radius of starlikeness (convexity) of $\frac{1}{2}(f(z)+g(z))$ is greater than $r_{0}$, where $r_{0}=0.3966 \ldots$ is the smallest positive root of the equation $1-3 r+2 r^{2}-$ $2 r^{3}=0$. Their method yields the same result when applied to the general convex combinations.

In this note, a sharp solution to Labelle and Rahman's problem is given. In fact, we make use of results of [3], [4] to prove that the radius of starlikeness of $F$ and that of the closed convex hull of starlike functions, $\overline{c o} \mathrm{St}$, are both equal to $r_{u}$.

Let $A_{0}$ be the set of functions $f(z)$ regular in $E$ and normalized by $f(0)=1$. Let $A_{1}$ be the set $\left\{z f: f \in A_{0}\right\}$. For any set $V \subseteq A_{1}$, we denote by $\hat{V}$ the set $\{f / z: f \in V\}$. The dual $U^{*}$ of any set $U \subseteq A_{0}$ is defined to be

$$
U^{*}=\left\{f \in A_{0}:(f * g)(z) \neq 0 \text { for all } g \in U, z \in E\right\},
$$

where $f * g$ is the Hadamard product of $f$ and $g$, i.e., if

$$
f(z)=\sum_{k=0}^{\infty} a_{k} z^{k}, \quad g(z)=\sum_{k=0}^{\infty} b_{k} z^{k}
$$

then

$$
(f * g)(z)=\sum_{k=0}^{\infty} a_{k} b_{k} z^{k}
$$

Received by the editors August 21, 1978.

AMS (MOS) subject classifications (1970). Primary 30A40, 30A32.

Key words and phrases. Radius of starlikeness, closed convex hull of starlike univalent functions, Hadamard product.

${ }^{1}$ This author acknowledges the financial support of a University of Tasmania Research Studentship. 
Also, by $U^{* *}$ we mean $\left(U^{*}\right)^{*}$. We then have

Lemma (RusCheWEYH [4]). Let $V, W \subseteq A_{0}$ and $g \in A_{0}$. Let

$$
\begin{aligned}
& r_{1}=\sup \left\{r:(g * f)(r z) \in V^{*} \text { for all } f \in W^{* *}\right\}, \\
& r_{2}=\sup \left\{r:(g * f)(r z) \in W^{*} \text { for all } f \in V\right\} .
\end{aligned}
$$

If $V$ is compact and if $f(x z) \in V$ for all $f \in V$ and $|x|<1$, then $\min \left(1, r_{1}\right)=$ $\min \left(1, r_{2}\right)$.

THEOREM. The radius of starlikeness of $\overline{\mathrm{co}}$ St is $r_{u}$, where $r_{u}=0.4035 \ldots$ is the positive root of the equation $r^{6}+5 r^{4}+79 r^{2}-13=0$.

Proof. Let $d(z)=\sum_{k=0}^{\infty}(k+1) z^{k}$, then for $f \in A_{0},(z f)^{\prime}=d * f$. Hence from Brickman, MacGregor and Wilken [1] we can write

$$
\widehat{\overline{\mathrm{CO} S t}}=\left\{d * f: f \in P_{1 / 2,0}\right\},
$$

where $P_{1 / 2,0}=\left\{f \in A_{0}: \operatorname{Re} f(z)>\frac{1}{2}, z \in E\right\}$. Thus the radius of starlikeness $r_{s}$ of $\overline{c o} \mathrm{St}$ is given by

$$
r_{s}=\sup \left\{r:(d * f)(r z) \in \widehat{\text { St }} \text { for all } f \in P_{1 / 2,0}\right\}
$$

Further, if we let

$$
\begin{aligned}
& P_{0}=\left\{f \in A_{0}: \exists \alpha \in \mathbf{R}, \operatorname{Re}\left\{e^{i \alpha} f(z)\right\}>0, z \in E\right\}, \\
& X=\left\{z \frac{1+\frac{1}{2}(\xi-\eta) z}{(1-\eta z)^{2}}:(|\eta|=|\xi|<1) \text { or }(\xi=\eta \text { and }|\xi|<1)\right\},
\end{aligned}
$$

then, from Roscheweyh [4], $P_{0}^{*}=P_{1 / 2,0}, P_{0}=P_{1 / 2,0}^{*}, \hat{X}^{*}=\widehat{\mathrm{St}}$ and $\hat{X}$ is compact with $f(x z) \in \hat{X}$ for all $f \in \hat{X},|x| \leqslant 1$. Therefore, from the Lemma, we find, on putting $V=\hat{X}, W=P_{1 / 2,0}$, that

$$
r_{s}=\sup \left\{r:(d * f)(r z) \in P_{0} \text { for all } f \in \hat{X}\right\},
$$

or equivalently,

$$
r_{s}=\sup \left\{r: f^{\prime}(r z) \in P_{0} \text { for all } f \in X\right\} .
$$

Since $X$ is a subset of $S$,

$$
r_{s} \geqslant \sup \left\{r: f^{\prime}(r z) \in P_{0} \text { for all } f \in S\right\} .
$$

Now, according to Robertson [3], there exists $\alpha \in \mathbf{R}$ such that $\operatorname{Re}\left\{e^{i \alpha f^{\prime}}(z)\right\}>$ 0 for $|z|<r, 0<r<1, f \in S$ if and only if, for every $\beta, h(z)=t f(z)+(1-$ $t) e^{-i \beta} f\left(e^{i \beta} z\right), 0<t<1$, is univalent in $|z|<r$; furthermore, $h(z)$ is univalent in $|z|<r_{u}$. Thus we have $r_{s} \geqslant r_{u}$. On the other hand, the radius of univalence of co St, which is also $r_{u}$, is greater than or equal to its radius of starlikeness $r_{s}$. Hence $r_{s}=r_{u}$.

The extremal function is as given by Robertson [3],

$$
f_{0}(z)=\frac{1}{2} \frac{z}{(1-z)^{2}}+\frac{1}{2} \frac{z}{\left(1-z e^{-2 i \alpha}\right)^{2}},
$$


where

$$
\cos \alpha=\frac{\left(1+3 r_{u}^{4}\right)^{1 / 2}+r_{u}^{2}-1}{2 r_{u}} .
$$

We note that since $f_{0}(z) \in F \subseteq \overline{c o}$ St, $r_{u}$ is also the radius of starlikeness of $F$.

\section{REFERENCES}

1. L. Brickman, T. H. MacGregor and D. H. Wilken, Convex hulls of some classical families of univalent functions, Trans. Amer. Math. Soc. 156 (1971), 91-107.

2. G. Labelle and Q. I. Rahman, Remarque sur la moyenne arithmétique de fonctions univalentes convexes, Canad. J. Math. 21 (1969), 977-981.

3. M. S. Robertson, The sum of univalent functions, Duke Math. J. 37 (1970), 411-419.

4. S. Ruscheweyh, Duality for Hadamard products with applications to extremal problems for functions regular in the unit disc, Trans. Amer. Math. Soc. 210 (1975), 63-74.

Department of Mathematics, University of Tasmania, Hobart, Tasmania, Australia (Current address of P. D. Tuan)

Current address (D. H. Hamilton): Department of Mathematics, Imperial College, London, England 